\title{
Walter Werbeck
}

\section{Glareans Vorstellung von modaler Stimmigkeit - Die für das Dodekachordon bestellten Kompositionen}

Welche Vorstellungen hatte Glarean von modaler Stimmigkeit? Was machte für ihn einen Modus aus? Welche Kriterien bestimmten ihn? Solche Fragen sind alles andere als leicht zu beantworten. Gewiss, Glarean konstruiert im zweiten Buch des Dodekachordon seine Tonarten penibel aus den sieben jeweils doppelt geteilten Oktavgattungen ${ }^{1}$. Doch muss er bei seinen Beispielen schon aus dem Cantus planus nur zu oft auf Ausnahmen von der Regel hinweisen, sei es, dass die jeweiligen Ambitus zu umfangreich oder zu gering ausfallen oder dass die Rahmentöne auf den falschen Positionen liegen. Er kommt deshalb nicht umhin, auch die jeweiligen Quint- und Quartgattungen zur Modusbestimmung heranzuziehen - freilich mit einigem Abstand zu den weitaus wichtigeren Oktaven. Erst im 37. Kapitel von Buch 2 geht er ausführlicher auf das Problem der Modi mit geringen Ambitus ein, und dementsprechend muss sich der Leser auch in Buch 3 bis Kapitel 25 gedulden, um »de tenoribus diapason non explentibus« (»Tenores, die die Oktave nicht ausfüllen «) $)^{2}$ informiert zu werden, über Ambitus also, bei denen die Quint- und Quartspezies Beachtung erfordern. Auch die jeweiligen Finales kommen erst bei schwierigen Ambitusverhältnissen ins Spiel.

An den Problemen, in die Glarean sich dennoch immer wieder verstrickt, ändert das wenig. Wie soll - um nur ein Beispiel aus dem Cantus planus zu nennen - der Leser mit der Tatsache fertig werden, dass der Dorius ebenso wie der Hypodorius nicht selten den Umfang des Hexachordum naturale, von $c$ bis $a$ also, einnehmen, ${ }^{3}$ sich demnach weder hinsichtlich des Ambitus noch der Finalis unterscheiden? Zwar wird ein Kenner schon gewusst haben, wie in solchen Fällen vorzugehen ist - bei Glarean aber findet er keine weitere Hilfestellung. Auch die traditionellen Repercussionsintervalle, die man in Deutschland mit Merkversen wie "Pri $r e-l a$, se $r e-f a$ etc. sich ein-

1 Dod, vor allem Buch 2, Kap. 2-6.

2 Dod., S. 430.

3 Dod., S. 171 findet sich ein einstimmiges Beispiel »Re la cum tono inferne, Dorii« (»für einen Dorius mit dem Ambitus d-a und Ganzton darunter «), auf S. 173 heißt es vom Hypodorius, er bewege sich »frequenter inter C \& G. ... adiecto superne tono ... (»Zwischen $\mathrm{c}$ und $\mathrm{g}$ mit einem Ganzton darüber $\ll$ ). In beiden Fällen handelt es sich also um den Ambitus des Hexachordum naturale, $c-a$. 
zuprägen pflegte, bleiben unerwähnt, obwohl Glarean eine solche »vulgaris agnitio« ("gewöhnliche Erkennungsweise») der Tonarten in Buch 1 des Dodekachordon immerhin noch mitgeteilt hatte. ${ }^{4}$

Selbst Glareans Allzweckwaffe, die "phrasis« der Tonarten, taugt nur ihm selbst als unfehlbares Kriterium für modale Stimmigkeit. Seinen heutigen Leser macht sie oft ratlos. Erstens hat der Schweizer Gelehrte, wie schon öfter bemerkt wurde, diesen Terminus nie genau definiert. ${ }^{5}$ Und zweitens hat in jüngster Zeit Frans Wiering mit Recht darauf aufmerksam gemacht, dass die Identifikation von "phrasis « mit dem Repercussionsintervall, wie sie Bernhard Meier ebenso wie Harold S. Powers ${ }^{6}$ vornahmen, nicht zutrifft - dies schon deshalb, weil sich in Buch 1 nicht etwa zwölf solcher Intervalle finden, sondern lediglich die üblichen acht (mit leichten Modifikationen). ${ }^{7}$ Glarean, so Wiering, habe "phrasis « vielmehr sehr viel weiter gefasst und als Merkmal für einen je individuellen modalen Stil verstanden. ${ }^{8}$ So sehr man Wiering beipflichten möchte: Eine Antwort auf die Frage nach Glareans Vorstellungen von modaler Stimmigkeit wird mit der Verallgemeinerung von "phrasis« nicht leichter.

Wiering beobachtete Glareans Umgang mit der "phrasis« in diesem umfassenderen Sinn vor allem im Zusammenhang mit den mehrstimmigen Modusanalysen. Offenbar sind die Kriterien von modaler Stimmigkeit im Cantus mensuralis noch schwieriger zu benennen als im Cantus planus. Schließlich werden dessen Gesetze, vor allem die der Solmisation, noch um die Regeln des mensuralen Rhythmus' sowie des Kontrapunkts vermehrt.

Man darf allerdings nicht zuviel verlangen. Ob die Modi überhaupt in die Satzlehre integriert werden müssten - über diese Frage waren sich nicht nur die Musiker des 16. Jahrhunderts uneins. Sie wird auch noch heute unterschiedlich beantwortet. Der Selbstverständlichkeit, mit der ein so profunder Quellenkenner wie Bernhard Meier behaupten konnte, den Kirchen-

4 Dod., Buch 1, Kap. 13, S. 32 f.

5 Dazu Bernhard Meier, "Heinrich Loriti Glareanus als Musiktheoretiker «, in: Beiträge zur Freiburger Wissenschafts- und Universitätsgeschichte 22 (1960), S. 93; außerdem zuletzt Frans Wiering, The Language of the Modes. Studies in the History of Polyphonic Modality, New York 2001 (Criticism and Analysis of Early Music, 3), S. 149-151.

6 Harold S. Powers, Art. "Mode", in: The New Grove Dictionary of Music and Musicians, hrsg. von Stanley Sadie, London 1980, Bd. 12, S. 409.

7 Dod., S. 32. Modifiziert ist das Intervall für den 5. Ton: Die übliche Quinte $-u t-s o l\left(f-b-c^{3}\right)$ bzw., in "pseudoklassischen " Texten, $f a-f a\left(f-q-c^{\prime}\right)$ - hat Glarean auf die Terz mi-sol reduziert, vermutlich um eine Verwechslung mit der $u t$-sol-Quinte des 7 . Tons auszuschließen.

8 F. Wiering, Language (wie Anm. 5), ebda. 
tonarten komme für die Kompositionspraxis der älteren Mehrstimmigkeit die gleiche Realität zu wie der Dur-Moll-Tonalität für die Musik späterer Zeiten, ${ }^{9}$ steht die gleichfalls quellengestützte Skepsis von Forschern wie Harold S. Powers und Jessie Ann Owens diametral gegenüber. ${ }^{10}$ Owens' Sicht auf "composers at work « im 16. Jahrhundert stützte sich zwar nur auf erhaltene Skizzen bzw. Entwürfe, machte aber eines mit bestürzender Klarheit deutlich: Die Kirchentonarten spielen beim Komponieren im Grunde keine Rolle. Allenfalls sekundär resultieren sie aus der Wahl eines der üblichen "tonal types ". ${ }^{11}$ Glarean und sein Dodekachordon sind denn auch für Owens' Buch ohne jede Bedeutung.

Der Schweizer Gelehrte entscheidet sich zwar, anders als sein Kölner Lehrer Johannes Cochlaeus oder der von ihm mehrfach zitierte Franchino Gaffurio, für die Einbeziehung der Mehrstimmigkeit in seine Moduslehre. Aber den Kontrapunkt behandelt er nicht und entzieht sich damit dem Problem einer konkreten Einbeziehung der Modi in die Satzlehre - warum sollte er auch mehr leisten als die musikalischen Spezialisten, die die Tonarten normalerweise nur in die Gesangslehre integrierten und sich beim Cantus figuratus auf Fragen der Mensuralnotation und manchmal auch des Kontrapunkts konzentrierten? ${ }^{12}$ Auch beschäftigt sich Glarean nicht wirklich mit den Konsequenzen der Solmisationslehre für die Tonarten und die Mehrstimmigkeit; ${ }^{13}$ wie üblich handelt er die poces musicales vor allem im Rahmen der Präliminarien von Buch 1 ab, und zwar in engem Anschluss an Gaffurio. Selbst die neuen Cantus durus und mollis werden von ihm nicht erwähnt. ${ }^{14}$ Dafür beginnt er Buch 3 mit mehreren Kapiteln zur Mensural-

9 Es genügt, in diesem Kontext auf Bernhard Meiers Hauptwerk zu verweisen: Die Tonarten der klassischen Vokalpolyphonie. Nach den Quellen dargestellt, Utrecht 1974.

10 Harold S. Powers, "Is Mode real? Pietro Aron, the Octenary System, and Polyphony", in: Basler Jahrbuch. für historische Musikpraxis 16 (1992), S. 9-52; Jessie Ann Owens, Composers at Work. The Craft of Musical Composition 1450-1600, New York 1997.

11 Dazu grundlegend Harold S. Powers, "Tonal Types and Modal Categories in Renaissance Polyphony«, in: Journal of the American Musicological Society 34 (1981), S. 428-470.

12 Zur Moduslehre zumal in deutschen Traktaten aus dem frühen 16. Jahrhundert vgl. Walter Werbeck, Studien zur deutschen Tonartenlebre in der ersten Hälfte des 16. Jahrbunderts, Kassel 1989 (Detmold-Paderborner Beiträge zur Musikwissenschaft, 1), insbes. S. 179-186.

13 Grundlegend zum engen Verhältnis zwischen Tonarten und Solmisationslehre ist nach wie vor Christian Bergers Buch Hexachord, Mensur und Textstruktur. Studien zum französischen Lied des 14. Jahrhunderts, Stuttgart 1992 (Beihefte zum Archiv für Musikwissenschaft, 35).

14 W. Werbeck, Studien (wie Anm. 12), S. 168f. 
lehre, damit die Laien unter seinen Lesern ${ }^{15}$ die mehrstimmigen Beispiele wenigstens einigermaßen verstehen konnten.

Die Modi bleiben für Glarean - ganz im Einklang mit seinen Zeitgenossen - auch in der Mehrstimmigkeit lineare Gebilde. Ahnlich wie zuvor Pietro Aron in seinem Trattato della natura et cognitione di tutti gli tuoni di canto figurato (Venedig 1525) nennt er mehrstimmige Kompositionen als Beispiele für die Existenz der Modi - besser: seiner Modi - auch im Cantus figuratus. Und um seine Thesen nachprüfbar zu machen, stellt er die Stimmen der jeweiligen Stücke jeweils wie in einem Chorbuch ${ }^{16}$ zusammen und druckt sie ab.

Die modalen Analysen im Dodekachordon sind nicht nur auf den jeweiligen Tenor konzentriert. Glarean verlässt sich nicht auf den Modus einer einzigen Stimme, wie er ja auch keineswegs allein mehrstimmige Bearbeitungen von Cantus firmi behandelt, die ihre Tonart schon mitbringen. Es geht ihm um den ganzen Tonsatz: ein Ziel, das er sich wohl erst im Laufe der Arbeit am Dodekachordon gesetzt hat. In seiner Motettensammlung jedenfalls, deren Vorwort 1527 niedergeschrieben wurde ${ }^{17}$, finden sich modale Bestimmungen oft nur für den Tenor; im Dodekachordon hingegen erstrecken sich die Analysen der aus der Sammlung übernommenen Stücke in der Regel noch auf weitere Stimmen: ${ }^{18}$ Zumal bei Kompositionen in den neuen Tonarten geht Glarean gründlich zu Werke. Und seine Beobachtungen zur "occulta cognatio ${ }^{19}$ (»verborgenen Verwandtschaft «) mancher Modi unterstreichen, wie sehr ihm daran gelegen ist, alle Stimmen in sein Konzept von Modalität zu integrieren.

15 Glarean wendete sich im Dodekachordon fraglos auch an Personen, die nicht nur Fachmusiker sein mussten. Vgl. dazu Laurenz Lütteken, »Humanismus im Kloster. Anmerkungen zu einem der Dedikationsexemplare von Glareans >Dodekachordon« «, in: Festschrift Klaus Hortschansky zum 60. Geburtstag, hrsg. von Axel Beer und Laurenz Lütteken, Tutzing 1995, S. 43-57.

16 Cristle Collins Judd, »Musical Commonplace Books, Writing Theory, and sSilent Listening:: The Polyphonic Examples of the Dodecachordon«, in: The Musical Quarterly 82 (1998), S. 507.

17 Universitätsbibliothek München, Sign. $8^{\circ}$ Cod. ms. 322-325 [= Cim 44 $\left.{ }^{2}\right]$ ). Vgl. zur Quelle allgemein Clytus Gottwald, Die Handschriften der Universitätsbibliothek München, Wiesbaden 1968 (Die Handschriften der Universitätsbibliothek München, 2), S. 70-75; zu den Beziehungen zum Dodekachordon vgl. Cristle Collins Judd, Reading Renaissance Music Theory. Hearing with the Eyes, Cambridge 2000 (Cambridge Studies in Music Theory and Analysis, 14), vor allem die Tabellen S. 142-146.

18 W. Werbeck, Studien (wie Anm. 12), S. 173, Anm. 200. Zu korrigieren sind hier die Angaben zu Antoine Brumels »Qui venit« und zu Josquins »Ave verum«: Glarean bestimmt im Dodekachordon nur die Tonarten von Cantus und Tenor, nicht aber des Bassus.

19 Dod., S. 251. Dazu H. Powers, Mode (wie Anm. 6), S. $410 f$. 
Die Schwierigkeiten, Glareans Vorstellungen von modaler Stimmigkeit $\mathrm{zu}$ benennen, werden damit freilich nicht geringer, sie potenzieren sich vielmehr. Hinzu kommt das Problem des Gesamtmodus. Wonach wird er ermittelt? Das Tenorprinzip greift zwar oft - wie zuletzt wiederum Frans Wiering betonte $-{ }^{20}$ aber eben nicht immer, ohne dass Glarean abweichende Fälle als Ausnahmen deklariert hätte, die eine Regel voraussetzten. Andererseits scheinen die Beziehungen etwa zwischen den a-, d- und e-Modi die Festlegung eines Gesamtmodus überhaupt zu erschweren bzw. zu modalen Familien von der Art der ut-, re- und mi-Tonarten zu führen, wie Glarean sie selbst gelegentlich erwähnt und Cristle Collins Judd in der Musik seiner Zeit beobachtet hat. ${ }^{21}$

Angesichts derart problematischer Verhältnisse darf man sich von einer Untersuchung der speziell für das Dodekachordon geschriebenen Kompositionen keine Wunder erwarten, aber doch vielleicht nähere Aufschlüsse über Glareans Konzept modaler Stimmigkeit - zumal in der Mehrstimmigkeit. Bevor ich auf diese Stücke näher eingehe, fasse ich kurz einige Fakten und darauf aufbauende Fragen wie Überlegungen zusammen.

1. Mit Sicherheit haben für das Dodekachordon Sixtus Dietrich und Gregor Meyer Kompositionen geliefert ${ }^{22}$ (vgl. die Übersicht in Tabelle 1 auf der folgenden Seite). Dietrich, der sich für die Musica speculativa interessierte, besaß als Vokalkomponist einiges Renommee, kaum jedoch der Solothurner Organist Meyer, von dem nahezu ausschließlich Beispiele für Glareans Buch nachweisbar sind. Meyer schrieb die meisten bestellten Stücke, darunter mit einem Kyrie als Beispiel für die Verbindung von Aeolius und Hypoaeolius eine der umfangreichsten Kompositionen im Dodekachordon über-

20 F. Wiering, Language (wie Anm. 5), S. 152.

21 Cristle Collins Judd, "Modal Types and $U t, R e, M i$ Tonalities: Tonal Coherence in Sacred Vocal Polyphony from about $1500 \ll$, in: Journal of the American Musicological Society 45 (1992), S. 428-467.

22 C. C. Judd, Commonplace Books, wie Anm. 16, S. 490, zufolge hat auch Ludwig Senfl sein "Deus in adiutorium meum indende $\alpha$ im lydischen Modus auf Bestellung geliefert. Belege dafür gibt es aber allem Anschein nach nicht. Wir wissen lediglich, dass Glarean Senfl vergeblich um das Kyrie de nostra Domina als Exempel für eine »mixtio Aeolii Hypoaeoliique bat. Siehe Eugen Tatarinoff (Hrsg.), Die Briefe Glareans an Johannes Aal, Stiftspropst in Solothurn, aus den Jahren 1538-1550, Solothurn 1895 (Urkundio, 2.3), S. 9 (Brief Glareans vom 10. Juli 1538). Dieses Kyrie lieferte dann Gregor Meyer. 
haupt. ${ }^{23}$ Vorstellbar wäre, dass sich Meyer besonders eng an Glareans Vorgaben klammerte, während Dietrich Theorie und Praxis souveräner zu verbinden wusste.

\begin{tabular}{|c|c|c|c|}
\hline Modus & $\begin{array}{l}\text { Ambitus/ } \\
\text { Struktur }\end{array}$ & $\begin{array}{l}\text { Titel (Textbeginn), ggf. Stimmen- } \\
\text { zahl }\end{array}$ & $\begin{array}{l}\text { Kompo- } \\
\text { nist }\end{array}$ \\
\hline Hyperaeolius & $H-f-b$ & O Domine Jesu Christe, 3-stg. & Dietrich \\
\hline Hypolydius & $c-f-c^{\prime}$ & Monas. Oberquintkanon ohne Text & Meyer \\
\hline Hypolydius & & Dyas. Ohne Text & Meyer \\
\hline Dorius & $d-a-d^{\prime}$ & Monas. Unterquintkanon ohne Text & Meyer \\
\hline Hypomixolydius & $d-g-d^{\prime}$ & Monas. Oberquintkanon ohne Text & Meyer \\
\hline Phrygius & $e-h-e^{\prime}$ & Monas. Unterquintkanon ohne Text & Meyer \\
\hline Lydius & $f-c^{\prime}-f^{\prime}$ & Forma inter $F$ \& e : Servus tuus, 2-stg. & Dietrich \\
\hline Lydius & & Tenor inter $F$ o $f$ : Erue Domine, 2-stg. & Dietrich \\
\hline Lydius & & Qui mihi ministrat, 4-stg. & Meyer \\
\hline Jonicus (auf $\mathrm{f}$ ) & $f-(\cong) c^{\prime}-f^{\prime}$ & Qui mihi ministrat, 4-stg. & Meyer \\
\hline Hyperphrygius & $f-h-f^{\prime}$ & Domine fac mecum, 4-stg. ad aequales & Dietrich \\
\hline Hyperphrygius & & Ab occultis, 4-stg. & Dietrich \\
\hline Hypoionisch & $g-c^{\prime}-g^{\prime}$ & Monas. Unterquintkanon ohne Text & Meyer \\
\hline $\begin{array}{l}\text { Hypoaeolius- } \\
\text { Aeolius }\end{array}$ & $e-a-e^{\prime}-a^{\prime}$ & $\begin{array}{l}\text { Kyrie I, IIa/b, III (je 4-stg.) } \\
\text { Christe I (4-stg.), II (3-stg.), III (4-stg.) } \\
\text { Kyrie I (4-stg.), II (3-stg.), IIIa/b (4-stg.) }\end{array}$ & Meyer \\
\hline 3. Quintspezies & $f-c^{\prime}(f a-f a)$ & Confitebor Domino, 4-stg. & Meyer \\
\hline
\end{tabular}

Tabelle 1: Übersicht über die bestellten Kompositionen von Gregor Meyer und Sixt Dietrich

23 Michele Calella, Art. "Meyer, Gregor«, in: Die Musik in Geschichte und Gegenwart, 2. Aufl., hrsg. von Ludwig Finscher, Personenteil, Bd. 12, Kassel und Stuttgart 2004, Sp. $119 \mathrm{f}$. 
2. Dietrichs und Meyers Beispiele dürften die neuesten mehrstimmigen Stücke für das Dodekachordon gewesen sein; sie entstanden erst Ende der 1530er bzw. Anfang der 1540er-Jahre, überwiegend wohl nach Abschluss des Textkorpus. ${ }^{24}$ Rückschlüsse auf eine besondere Modernität der Musik wären jedoch voreilig. Dietrichs Stücke sind seine letzten Kompositionen überhaupt ${ }^{25}$ und wie er sich im Wesentlichen am Stil der Josquin-Generation orientierte, so auch der weitaus jüngere Gregor Meyer. Von einem Schritt in die Richtung der so genannten klassischen Vokalpolyphonie, wie ihn beispielsweise Meyers Generationsgenosse Jacobus Clemens non Papa tat, ist nichts zu spüren. In das Korpus der Kompositionen des Dodekachordon passen sich die bestellten Beispiele bruchlos ein.

3. Die meisten der bestellten Beispiele gehören den Tonarten an, für die Glarean in der ihm zur Verfügung stehenden Literatur keine oder nur wenige Stücke fand (in Tabelle 1 grau unterlegt): die Modi rejecti, $\mathrm{Hy}$ perphrygius und Hyperaeolius (drei Beispiele), sowie der "alte" Lydius bzw. Hypolydius (fünf Beispiele, dazu ein sechstes für die Nähe des Lydius zum Jonicus sowie, als siebtes, Meyers "Confitebor Domino" zur mehrstimmigen Demonstration der Quinte $f a-f a)$. Zu diesen Tonarten hinzu kommt die schon genannte Verbindung von Aeolius und Hypoaeolius in Meyers Kyrie de nostra Domina, das Glarean zufolge sonst stets in verderbter Form bearbeitet wurde. ${ }^{26}$ Bei den restlichen vier Beispielen handelt es sich um Kanons zu gebräuchlichen Modi: Dorisch, Hypomixolydisch, Phrygisch und Hypoionisch.

4. Glarean traute - wenig überraschend - Dietrich mehr zu als Meyer. Jener lieferte ausschließlich Beispiele für schwierigere Tonarten: die Modi rejecti (ein Trio, zwei vierstimmige Stücke) sowie zwei Duos zum Hypolydius. Meyer dagegen steuerte, abgesehen von drei Beispielen zu den alten fModi, nur Stücke in verbreiteten Tonarten bei. Außerdem lieferte er sämtliche Monaden (zweistimmige Kanons), also die einfachsten Beispiele, mit denen Glarean (nach dem Vorbild von Sebald Heydens Musicae, id est artis

24 Zur Chronologie des Dodekachordon vgl. Franz-Dieter Sauerborn, "Michael Rubellus von Rottweil als Lehrer von Glarean und anderen Humanisten. Zur Entstehungsgeschichte von Glareans Dodekachordon «, in: Zeitschrift für Württembergische Landesgeschichte 54 (1995), S. 61-75, vor allem S. 69-75. Über seine Wünsche an Meyer korrespondierte Glarean mit Aal in den Jahren 1538 und 1540; vgl. E. Tatarinoff, Die Briefe (wie Anm. 22), S. 15-23.

25 Hermann Zenck, Sixtus Dietrich. Ein Beitrag zur Musik und Musikanschaunng im Zeitalter der Reformation, Leipzig 1928, Reprint Hildesheim 1967 (Publikationen älterer Musik, 3.2), S. 46.

26 Dod., S. 366. 
canendi libri duo von 1537$)^{27}$ jeweils zu beginnen pflegte. Aber Meyer schrieb auch vier vierstimmige Stücke.

5. Das größte Hindernis für eine adäquate Bewertung der bestellten Kompositionen liegt darin, dass wir kaum etwas darüber wissen, wie weit Glarean - abgesehen von seiner Theorie - konkret auf die jeweiligen Stücke eingewirkt hat. Clement A. Miller hat im Vorwort zu seiner Übersetzung des Dodekachordons das wenige zusammengefasst, was sich erschließen lässt: ${ }^{28}$ Dietrich war vermutlich gehalten, für den Lydius jeweils ein Beispiel mit dem Tenorambitus einer vollständigen und einer um ein Semitonium reduzierten f-Oktave zu schreiben. Außerdem dürfte er von Glarean dessen einstimmiges Beispiel für den Hyperaeolius, "O Domine Jesu Christe«, als Grundlage für seine dreistimmige Bearbeitung erhalten haben. Und vermutlich hat Glarean ihn auch auf das Offertorium »Domine, fac mecum « als Cantus firmus für eine hyperphrygische Komposition aufmerksam gemacht. $\mathrm{Zu}$ vermuten ist ein ähnliches Procedere für das in Buch 2 abgedruckte $K y$ rie de nostra Domina sowie die Communio "Qui mihi ministrat « als Basis für die jeweiligen Bearbeitungen Gregor Meyers. Das sind insgesamt nicht sehr viele Informationen, und man wüsste gerne mehr.

Was hat Glarean mit Dietrich und Meyer besprochen? Worauf sollten sie achten? War ihm eher an der Demonstration seiner Theorie oder eher an der Praktikabilität der Exempla gelegen, oder sollten beide Gesichtspunkte zu gleichen Teilen berücksichtigt werden? Sodann: Wer war es, der bei den Monaden das Kanonintervall, bei den Dyaden die jeweils gewählten Stimmlagen festlegte? Kannten Dietrich und Meyer die übrigen von Glarean ausgewählten Kompositionen? Waren sie vielleicht sogar gehalten, sich an deren Schreibart zu orientieren? Schließlich: Hat es Revisionen gegeben? Verlangte Glarean Änderungen - zugunsten theoretischer Eindeutigkeit oder vielleicht auch zugunsten der Gesangspraxis? Musste er aus Zeitgründen Stücke in Kauf nehmen, auf die er gerne verzichtet hätte? War er wirklich so begeistert von Meyers Stücken, wie er in seinen Briefen an Johannes Aal schrieb? Zur Vorsicht jedenfalls mahnt eine Formulierung vom November 1538: er, Glarean, sei nicht in der Lage, Meyers eingereichte Stücke zu beurteilen. ${ }^{29}$ Zählte Meyers Kyrie nur als Notlösung, als Ersatz für die er-

27 C. C. Judd, Reading Renaissance Music Theory (wie Anm. 17), S. 139 und 158-170.

28 Clement A. Miller, Heinrich Glarean, Dodecachordon. Translation, Transcription and Commentary, Rom 1965 (Musical Studies and Documents, 6), »Introduction «, Bd. 1, S. 30.

29 E. Tatarinoff, Die Briefe (wie Anm. 22), S. 15: „... non sum tanti iudicii, ut utram de binis compositionibus eligam sciam ...«. 
hoffte, aber nicht erhaltene Senfl'sche Komposition? ${ }^{30}$ Antworten auf alle diese Fragen gibt es nicht; ob die bestellten Stücke in jeder Hinsicht Glareans Intentionen entsprachen, muss vorerst offen bleiben.

Ich beginne meine Untersuchung mit einigen der Meyer'schen zweistimmigen Kanons. Dazu zunächst eine kurze Übersicht.

\begin{tabular}{l|l|l|c|l} 
Tonart & Kanonintervall & $\begin{array}{l}\text { Tonart im Abstand } \\
\text { des Kanonintervalls }\end{array}$ & $\begin{array}{l}\text { cogna- } \\
\text { tio }\end{array}$ & Hexachorde \\
\hline Hypolydius & Oberquinte & Hypojonicus & + & $\begin{array}{l}\text { naturale } \rightarrow \text { du- } \\
\text { rum }\end{array}$ \\
Dorius & Unterquinte & Mixolydius & - & naturale $\rightarrow$ molle \\
Hypomixolydius & Oberquinte & Hypodorius & - & $\begin{array}{l}\text { naturale } \rightarrow \text { du- } \\
\text { rum }\end{array}$ \\
Phrygius & Unterquinte & Aeolius & + & $\begin{array}{l}\text { naturale } \rightarrow \text { molle } \\
\text { durum } \rightarrow \text { natura- }\end{array}$ \\
Hypojonicus & Unterquinte & Hypolydius & +
\end{tabular}

Tabelle 2: Die von Gregor Meyer für das Dodekachordon komponierten Kanons ${ }^{31}$

Im Kontext des berühmten Schlusskapitels »de Symphonetarum ingenio" bemerkt Glarean zu Kanons im Sekundabstand, in solchen Stücken stießen die "Systemata binorum Modorum « zusammen. ${ }^{32}$ Auch seine Analysen von Stimmen mit Ambitus im Quintabstand führen manchmal zur Ermittlung jeweils verschiedener Modi: Eine a-Oktave ist ein Aeolius, eine e-Oktave hingegen ein Phrygius. ${ }^{33} \mathrm{Im}$ Zusammenhang mit Meyers Quintkanons hin-

30 Siehe oben Anm. 22.

31 Angegeben sind: die Tonart, die der Kanon im Dodekachordon repräsentiert (Spalte 1), der Intervallabstand zur 2. Stimme (Spalte 2) und die durch die 2. Stimme repräsentierte Tonart (Spalte 3). Spalte 4 gibt Auskunft darüber, ob es sich um zwei enger verwandte Tonarten gemäß Glareans Ausführungen zur »occulta cognatio“ (siehe oben Anm. 19) handelt $(+)$ oder nicht $(-)$, und aus Spalte 5 lassen sich die in den jeweiligen beiden Kanonstimmen dominierenden Hexachorde ablesen.

32 Dod., S. 446.

33 Zum vierstimmigen "Tulerunt Dominum» als Exempel für den Phrygius schreibt Glarean: "In ea cantione Tenor ac Altitonans Phrygium Modum elegantissime arsi ac Thesi habent. Basis autem ac Cantus Aeolium ....« (»In diesem Stück prägen Tenor und Alt den 
gegen spricht Glarean nie von zwei verschiedenen Modi - obwohl doch auch hier aus der Oktave eines Modus stets die Oktave eines anderen resultiert, seien es nun Tonarten mit einer occulta cognatio oder nicht. Bestand also die Aufgabe für Meyer darin, einen Modus lediglich einstimmig (mit beliebigem Kanonabstand) oder auch im genau kalkulierten Stimmenverbund eines zweistimmigen Quintkanons zu realisieren? Verdeutlichen die scheinbar so einfachen Kanons mehr als nur ein lineares Moduskonzept?

Bejahen könnte man diese Frage beim hypojonischen Kanon; Glarean bemerkt hier ausdrücklich, der Modus werde in beiden Stimmen dargestellt. ${ }^{34}$ Das kann bedeuten: Jede Stimme prägt für sich die Tonart aus. Das kann aber auch bedeuten: Beide Stimmen wirken bei der Darstellung des Modus zusammen. Und Meyers Kanon vermittelt eher letzteren Eindruck (siehe Notenbeispiel $\mathbf{1}$; in Klammern die jeweiligen Intervalle der Kanonstimme). Der Organist hat (wohl auch, um eine Vermischung mit dem in
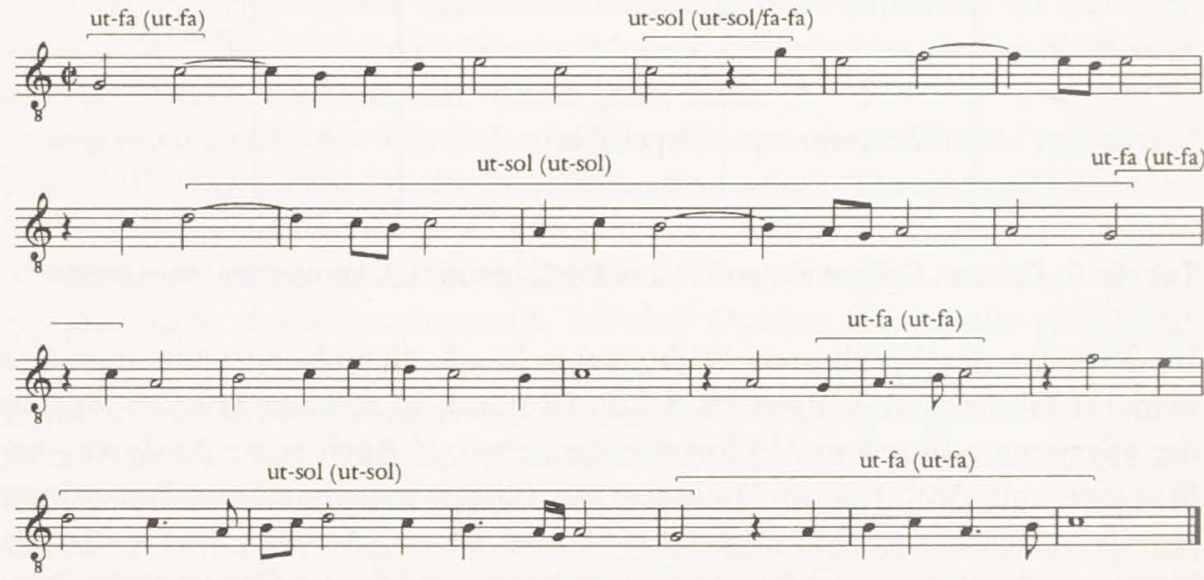

Notenbeispiel 1: Gregor Meyer, Monas im Hypojonicus (Unterquintkanon)

der Unterquinte stehenden Hypolydius zu vermeiden) die Kanonmelodie wesentlich durch solche modustypischen Intervalle strukturiert, die in der Unterquinte gleich bleiben, somit auch gleich solmisiert werden können: die Quarte $u t-f a$ in den Positionen $g-c^{\prime}$ (bzw. im Kanon $c-f$ ), und die Quin-

phrygischen Modus sehr elegant aus, und zwar in auf- und absteigender Bewegung. Bass und Sopran hingegen haben den Aeolius «.): Dod., S. 312:

34 Dod., S. 354: »... utraque uox Modum referat." Vgl. Übs. Miller, Bd. 2, S. 433. 
te $u t-s o l$ in den Positionen $g-d^{\prime}$ ' (bzw. im Kanon $c-g$ ). Mit anderen Worten: Die hypojonische Quarte $g-c^{\prime}$ ( $u t-f a$ ) prägt die Ausgangsstimme, die hypojonische Quinte $c-g$ (ut-sol) hingegen die Unterquintstimme. Beide ergänzen sich zur Modusdarstellung. Die Gefahr einer Kollision der lydischen Quinte $f a-f a$ mit der jonischen $u t$-sol wird vermieden, weil die $u t$-sol-Quinte in der Ausgangsstimme nahezu immer gerade nicht in der (eigentlich zu erwartenden) jonischen Position, sondern in der mixolydischen erscheint. Offensichtlich hat nicht nur der Komponist, sondern auch Glarean dieses Manko in Kauf genommen.

Ähnlich ist Meyer im hypomixolydischen Kanon vorgegangen ${ }^{35}$ (siehe Notenbeispiel 2). Allerdings führt hier die Konzentration auf oberquintidentische Quarten und Quinten zu schwerwiegenden Konsequenzen für den Modus, um den es geht: Weil Meyer den Konflikt zwischen der mixolydischen $u t$-sol-Quinte und der dorischen $r e-l a$-Quinte verhindern will, verkürzt er die hypomixolydische d-Oktave um den obersten Ton, verzichtet also auf die $u t-$ sol-Quinte und pointiert stattdessen mehrfach die re-la-Quinte. Sein Beispiel im Hypomixolydius könnte folglich über weite Strecken ebenso gut den Dorius repräsentieren. Glarean hat sich jeden Kommentars enthalten.
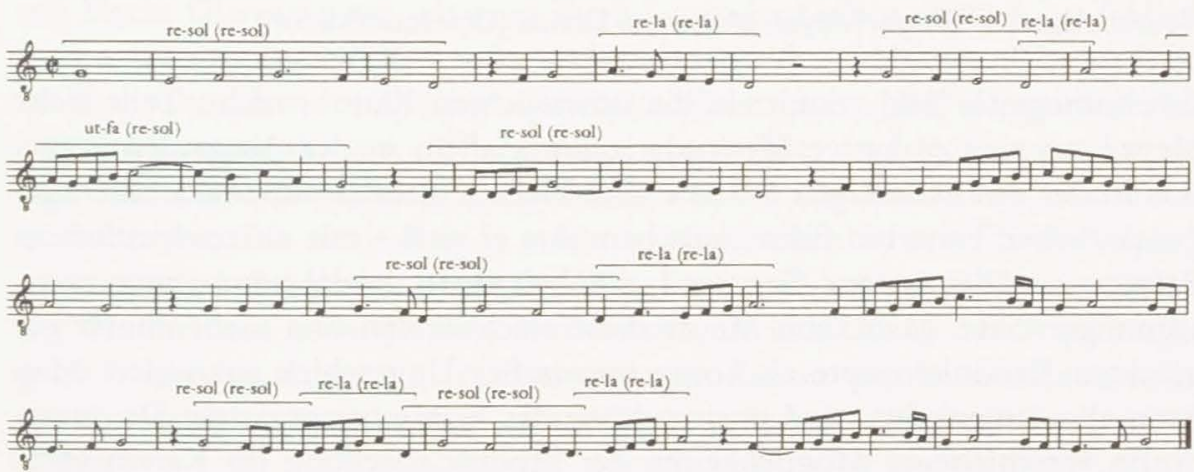

Notenbeispiel 2: Gregor Meyer, Monas im Hypomixolydius (Oberquintkanon)

Hingegen lobte er ausdrücklich ${ }^{36}$ den schönen dorischen Kanon Meyers - obwohl gerade hier von einer beide Stimmen integrierenden Modusdarstellung kaum die Rede sein kann (siehe Notenbeispiel 3 auf der folgenden Seite).

35 Dod., S. 304; Übs. Miller, S. 379.

36 Das sei ein Stück, "Vbi bellissime Modus inter D et d decurrit.« (»wo der Modus aufs schönste zwischen $d^{\prime}$ und $d$ verläuft.«): Dod., S. 296; Übs. Miller, S. 368. 
Die Wahl der Unterquinte als Kanonintervall (Dorius $\rightarrow$ Mixolydius) signalisiert wiederum eher differierende als identische Intervallgattungen; und in der Konsequenz prägt sich, anders als zuvor, der Dorius in der Ausgangsstimme ebenso deutlich aus wie der Mixolydius in der Kanonstimme. Man fragt sich, warum Meyer in diesem Fall nicht einen Oberquintkanon geschrieben und auf die cognatio zwischen Dorius und Aeolius gesetzt hat. ${ }^{37}$

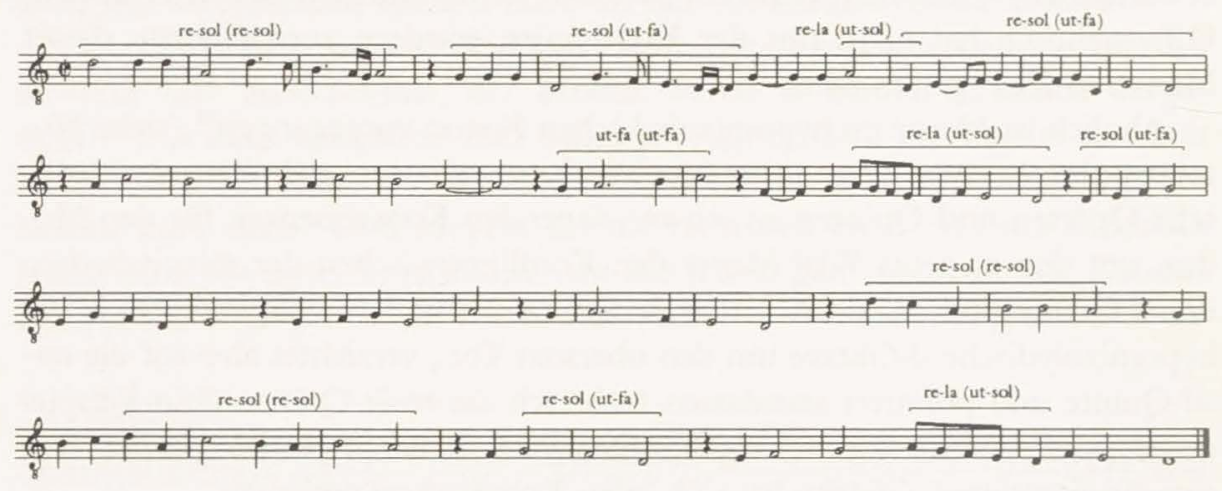

Notenbeispiel 3: Gregor Meyer, Monas im Dorius (Unterquintkanon)

Ein homogenes Bild vermitteln die untersuchten Kanons nicht. Teils zielte Meyer auf eine plakative Modusdarstellung allein in der Ausgangsstimme, was in der zweistimmigen Summe zum Nebeneinander außerordentlich gegensätzlicher Tonarten führt, teils bemühte er sich - mit unterschiedlichem Erfolg - um das genaue Gegenteil, nämlich einen modal homogenen zweistimmigen Satz. Man kann Meyer diese wechselnden und nicht immer geglückten Kanonkonzepte als kompositorisches Ungeschick ankreiden. Man kann aber umgekehrt, und positiv gewendet, von einer gezielten Demonstration verschiedener Möglichkeiten der Modusdarstellung im Kanon sprechen, die Meyer vielleicht in enger Absprache mit Glarean vorgenommen hat. Dieser wäre dann keineswegs nur an einer einstimmigen, eng an den jeweiligen Intervallspezies orientierten modalen Präsentation interessiert gewesen, sondern hätte auch eine differenzierte, die Grenzen einer Stimme

37 Überhaupt stellt sich die Frage, warum Meyer gleich dreimal das Unterquintintervall wählte, obwohl damit jedesmal Schwierigkeiten verbunden waren: entweder (s. Tabelle 2) der Wechsel vom Hexachordum naturale ins Hexachordum molle (mit dem für Glarean inakzeptablen Nebeneinander von b-rotundum und $q$-quadratum oder, beim hypoionischen Kanon, der Wechsel von der jonischen zur lydischen Quinte. 
überwindende Modusdarstellung geschätzt. Meyer jedenfalls konnte schon an diesen einfachen Exempeln zeigen, dass er auch in mehr als nur einer Stimme modal zu denken verstand.

Der hypolydische Kanon Meyers ${ }^{38}$ fällt aus dem Rahmen. Er repräsentiert einen der Modi, für die Glarean in der Literatur nur wenige Beispiele gefunden hatte. Lydius und Hypolydius gehören - im Gegensatz zu allen anderen zehn Tonarten - gerade nicht zu den gebräuchlichen Modi; Glarean musste sie, so seine Klage, aus dem Jahrhunderte alten Schutt falscher Traditionen erst wieder freilegen. Mehrstimmige lydische bzw. hypolydische Stücke zu schreiben bedeutete, sich mit einem Problem auseinander zu setzen, dessen große Tragweite bislang die Existenz von Glareans Lydius nahezu unmöglich gemacht hatte: der 3. Quintgattung $f a-f a$, deren Lebensfähigkeit angesichts der Realität des Hexachordum molle in der Gesangs- wie Kompositionspraxis mehr als gering war.

Im Grunde hatte Meyer keine Chance, mit seiner Kanonstimme diese verquere Quinte zu etablieren. Vielmehr bestätigt er - wohl unfreiwillig -, wie Recht Glarean hatte, als er in Buch 1 vor der Gefahr warnte, bei f-Modi in den Jonicus zu verfallen, oder anders gesagt: den f-Modus nicht im Cantus durus, sondern im üblichen Cantus mollis vorzutragen. ${ }^{39}$ Das musste bei einem Oberquintkanon, wie Meyer ihn schreibt, erst recht nahe liegen, denn nicht über $h$ - $m i$, wohl aber über $b$-fa stellte das Tonsystem eine Oberquinte bereit. Die clavis $h-m i$ in seiner hypolydischen Stimme dürfte folglich meistens als $b$-fa solmisiert worden sein, und auch bei einem Durchgang $a-$ $b-c$ ' konnte man $r e-m i-f a$ singen, ohne sich die lydische Quinte $f a-f a$ vergegenwärtigen zu müssen. (Der Einwand, der Kanon sei, da untextiert, nicht vokal, sondern instrumental auszuführen, ändert an dem Sachverhalt nichts.)

Meyer kann sich dem Dilemma nur durch halbherzige Lösungen entziehen: $\mathrm{Zu}$ Beginn lässt er dem geforderten $h$-mi beispielsweise gerne die Untersekunde $a$-re vorausgehen; im enggeführten Quintkanon wird daraus die Folge $e-f$, so dass $h$ (in der Ausgangsstimme) und $e$ (in der Kanonstimme) als Quarte $m i-m i$ verstanden werden können. Eindämmen ließ sich so die molle-Solmisation kaum - zumal dann nicht, wenn die clavis über $\boldsymbol{a}$-la nicht 
direkt zum $c$ aufwärts, sondern wie ein $f a$-sopra-la erneut abwärts geführt wird. Meyer verzichtet denn auch in der zweiten Hälfte seines Kanons fast vollständig auf die kritische Stufe über $a$ - zu Lasten freilich der hypolydischen Identität.

Das grundsätzliche Problem, die Ausführung der lydischen Quinte nicht durch das nahe liegende Hexachordum molle, sondern durch die Hexachorde naturale und durum inklusive Mutation, bleibt in allen lydischen bzw. hypolydischen Exempeln eine Herausforderung für die Komponisten. Meyer hat sie vor allem in seinem hypolydischen Duo bestanden. ${ }^{40}$ Die maßgebliche Quinte $f a-f a$ wird nämlich, vor allem zu Beginn und am Schluss, in beiden Stimmen gewissermaßen maskiert, und zwar als durch einen zusätzlichen Ganzton ergänzte Modusquarte $u t-f a\left(g-c^{\prime}\right)$. Innerhalb dieser Quarte aber - sie erscheint selbstverständlich auch im Hexachordum naturale zwischen den claves $c$ und $f$-nimmt $h$-mi einen normalen Platz ein. Vielleicht hob Glarean in seinem Kommentar gerade deshalb die Einhaltung des hypolydischen »systema naturale « auch in der Oberstimme hervor (und verschwieg ihre durch die doppelte Quarte $u t-f a$ verursachte Nähe zum Hypomixolydius $).^{41}$ Meyers Beispiel taugt jedenfalls für mehr als zur bloßen Illustration einer Theorie: Es ist auch ein Stück aufführbare Musik.

Gleiches ließe sich von Sixt Dietrichs lydischen Duos behaupten. ${ }^{42}$ Im ersten fehlt, wohl auf Grund einer Vorgabe Glareans, ${ }^{43}$ der modusprägenden Tenorstimme das obere $f$ und damit die Möglichkeit einer Kadenz in dieser hohen Lage. Vielleicht deshalb hat Dietrich generell auf deutliche Zäsuren, zumal auf der Finalis, verzichtet und sich dafür entschieden, den Modus bzw. dessen prekäre Quintspezies durch einen gerade nicht an den lydischen Intervallen orientierten Bass abzusichern. Gleich zu Beginn erklingt hier in mehrfacher Wiederholung die $r e-l a$-Quinte $d-a$, die der Tenor zur vollständigen d-Skala auffüllt - weshalb für eine Solmisation der 6. Stufe als $b$-fa kein Anlass besteht. Auf dieser Basis kann Dietrich später sogar eine Sequenz schreiben, die im Bass eigentlich zu $b$ - $f a$ (Unterquinte zum $f$ $f a$ im Tenor) führen müsste, erstickt einen drohenden modalen Flächenbrand jedoch rasch durch den Wechsel vom Quint- in den Oktavkanon (sie-

40 Dod., S. 281; Übs. Miller, S. 352 f.

41 Im 8. Kirchenton spielt das Repercussionsintervall $u t-f a$ seit jeher eine wesentliche Rolle.

42 Dod., S. 328f.; Übs. Miller, S. 407f.

43 Glarean hatte schon in Kap. 25 des 2. Buches (Dod., S. 128) darauf hingewiesen, der Lydius sei bei den Alten häufig entweder mit vollständiger Oktave oder mit oben um einen Ton verkürzter Oktave verwendet worden. Deshalb schreibt Dietrich zwei Duos: das eine zur Demonstration der »forma inter $F$ \& $e$ «, das zweite für den »Tenor inter $F$ \& $f$ « (Dod., S. 328f.). 
he Notenbeispiel 4). Dietrichs Konzept, die Unterstimme auf die Fundierung des Lydius bzw. der lydischen Quinte in der Oberstimme zuzuschneiden, führt zu einem durchaus praktikablen Resultat, doch fällt ihm eine eigene lydische oder hypolydische Identität im Bass zum Opfer. Dessen Tonart wird von Glarean denn auch nicht weiter bestimmt.

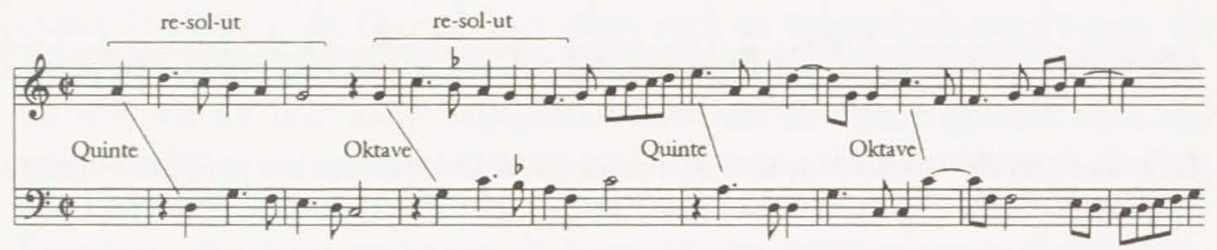

Notenbeispiel 4: Sixt Dietrich, Ausschnitt aus dem Duo »antiqui Lydii Forma inter F \& e«

Über Gregor Meyers lydisch-jonische Doppelvertonung des »Qui mihi ministrat $^{44}$ hat sich zuletzt Harold Powers geäußert. Seine Beurteilung der lydischen Version - es handle sich um nichts mehr als "made-up music purporting to illustrate the meaning of Glarean's real theory ${ }^{45}$ - lässt sich noch zuspitzen. Nicht nur die lydische, auch die jonische Variante ist weniger "real music" als vielmehr Illustration von Glareans Behauptung der großen Nähe zwischen den beiden Modi. Weil das Postulat ebenso wie der stets gleiche Cantus firmus vorgegeben war, achtete Meyer darauf, seine Kompositionen möglichst ähnlich zu halten und die systembedingten Unterschiede - vor allem die Möglichkeit oder Unmöglichkeit von Klängen auf der 4. Stufe - eher zu unterdrücken. Das ist ihm fraglos gelungen. Die Klangtechnik differiert über weite Strecken kaum; auch deshalb, weil Meyer in der jonischen Variante immer wieder mit der Wendung $c$ '"- $b$ '-c" bei gleichzeitiger Sext-Oktav- oder Terz-Quint-Progression arbeitet, mit einer typischen Klausel also, die normalerweise in der Oberstimme einen Halbtonanschluss impliziert: Gesungen wird nicht die notierte Folge $c$ "- $b$ '-c", sondern $c^{\prime \prime}-h^{\prime}-c$ " (siehe Notenbeispiel $5 \mathrm{a}$ auf der nächsten Seite). Je häufiger aber ein derart akzidentelles $h$ auftritt, desto geringer die Differenz zum lydischen Beispiel. Aus der Musik lässt sich der vorgeschriebene Cantus durus oder mollis noch nur selten ermitteln. Allein das jonische Supplement (siehe Notenbeispiel $5 c$ auf der nächsten Seite; zum Vergleich der lydische Schluss

44 Dod., S. 338-341; Übs. Miller, S. 416-421.

45 Harold S. Powers, "Music as Text and Text as Music «, in: Musik als Text. Bericht über den Internationalen Kongre $\beta$ der Gesellschaft für Musikforschung Freiburg im Breisgau 1993, hrsg. von Hermann Danuser und Tobias Plebuch, Kassel 1998, Bd. 1, S. 25. 
in Notenbeispiel 5d) mit seinem betonten Klang auf $B$ macht eine Ausnahme - und wirkt deshalb fast wie ein Fremdkörper.

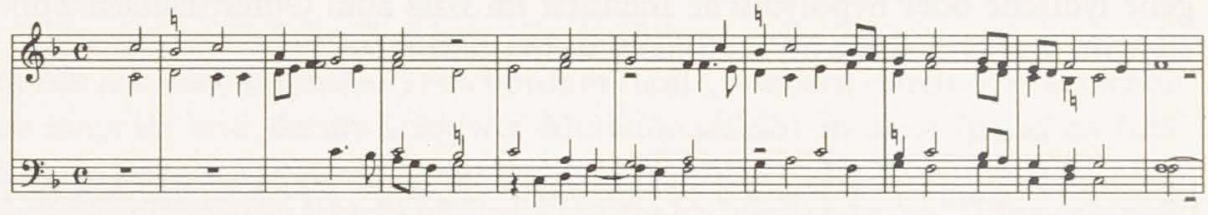

Notenbeispiel 5a: Gregor Meyer, »Qui ministrat«, Ausschnitt aus der jonischen Version (Cantus mollis)

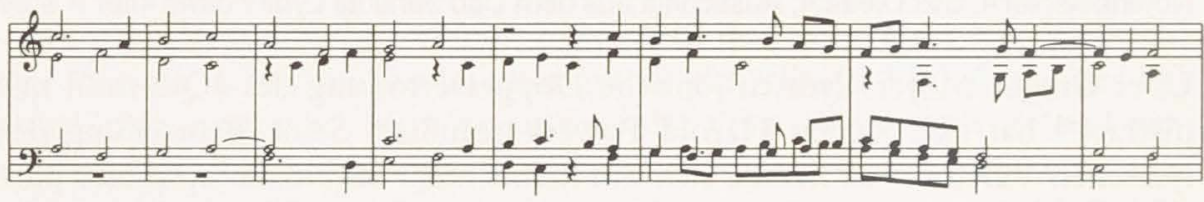

Notenbeispiel 5b: Gregor Meyer, »Qui ministrat«, Ausschnitt aus der lydischen Version (Cantus durus)

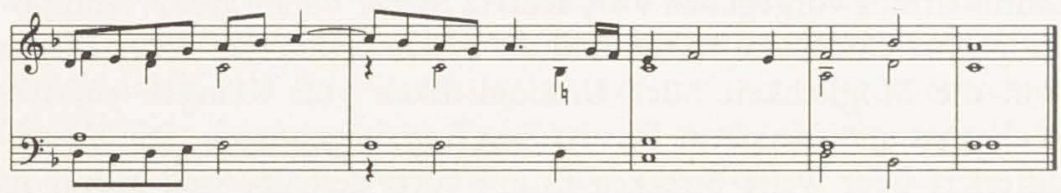

Notenbeispiel 5c: Gregor Meyer, "Qui ministrat", Schluss der jonischen Version

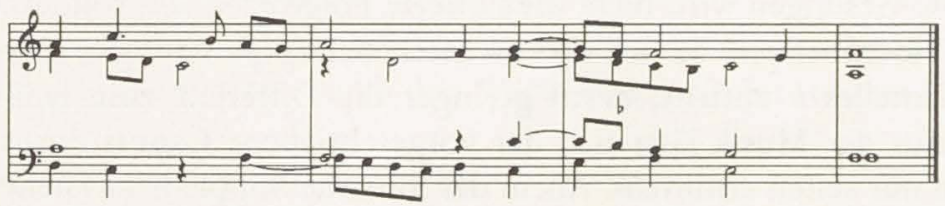

Notenbeispiel 5d: Gregor Meyer, "Qui ministrat«, Schluss der lydischen Version 
Erst recht gänzlich Glareans Doktrin geschuldet sind die Beispiele für die beiden Modi rejecti, den »authentischen " Hyperaeolius mit der Oktave $H-f$ $h$ und den "plagalen " Hyperphrygius mit der Oktave $f-h-f$ ". Die Entscheidung des Schweizer Gelehrten, auch für solche Modi Exempel vorzulegen, entbehrt nicht einer gewissen Gewaltsamkeit. Weil wichtige modale Kriterien nicht oder nur eingeschränkt funktionieren - es gibt keine QuintQuart-Struktur -, ist Glarean hier denn auch zu Zugeständnissen bereit. So schlägt er schon in Buch 2 für den Hyperaeolius Modifikationen des Ambitus vor, wie sie auch sonst begegneten: etwa die Erweiterung nach oben um einen Halbton und nach unten um einen Ganzton. Ein Ambitus $A-c^{\prime}$ nämlich ließe sich, zumindest für ein »ingenium exercitatum « (»einen versierten Kenner «), durchaus realisieren. ${ }^{46}$ Vom Modus bleiben unter diesen Umständen allein das für Glarean nicht verhandelbare "Systema«, also der Cantus durus, sowie die Töne $h-m i$ und $f$-fa erhalten. Sie spielen in der Melodik eine Rolle, und $h-m i$ ist die Finalis. Die falschen Intervalle hingegen, die den Hyperaeolius ebenso wie den Hyperphrygius überhaupt erst zu Modi rejecti machen und sie aus dem Kanon der zwölf Tonarten ausschließen, spielen in den Beispielen keine oder allenfalls eine untergeordnete Rolle. Mit anderen Worten: Ein ein- wie mehrstimmig komponierter Modus rejectus ist nichts weniger als eine klassische contradictio in adiecto, weil jegliche praxistaugliche, die fehlende Quint-Quart-Struktur umschiffende Realisierung seinen Status als rejectus wieder aufhebt. ${ }^{47}$ Kein Wunder, dass Glarean für einen solchen Modus so gut wie keine mehrstimmigen Beispiele gefunden hat. ${ }^{48}$

Unter diesen Umständen dürften sich für Dietrich die Schwierigkeiten bei seiner dreistimmigen Komposition des Hyperaeolius in Grenzen gehalten haben. Ein von Glarean bereitgestellter ${ }^{49}$ Cantus firmus mit dem Dezimenambitus $A-c^{\prime}$ (von Dietrich auf $A-b$ reduziert), den Haupttönen $b$

46 Dod., S. 112.

47 Dass die Modi rejecti Glarean zur Stärkung seiner These von der Existenz der zwölf Modi im ein- wie mehrstimmigen Repertoire dienen, hat Sarah Fuller betont: »If even modes rejected on theoretical grounds occasionally appear in plainchant and in some sacred polyphony, then surely the twelve properly constituted modes must have a secure place in these repertories.« (»Defending the Dodecachordon: Ideological Currents in Glarean's Modal Theory «, in: Journal of the American Musicological Society 49, 1996, S. 214.) Das ändert freilich nichts an der Absurdität eines praktikablen »rejectus«, weil eben die Praxistauglichkeit mit seinem Status unvereinbar ist.

48 "Huius [sc. modi] quidem integrum nullum, quod sciam, invenitur ... (»Von diesem Modus findet man meines Wissens kein vollständiges Beispiel«): Dod., S. 276

$49 \mathrm{Er}$ ist in Buch 2 bereits abgedruckt worden. Vgl. Dod., S. 113. 
und $f$ sowie einer Strukturierung primär durch die Quinte $r e-l a$ und die Quarte mi-la: Das musste in $\gg \mathrm{O}$ Domine Jesu Christe ${ }^{50}$ geradezu zwangsläufig zu einer Mischung von Modi mit den Finales $e$ und $a$ führen, die denn auch in allen Stimmen zu beobachten ist. ${ }^{51}$ Zusätzlich rückt Dietrich auch die falschen Hauptintervalle ins Licht, bettet deren Töne aber konsequent in dreistimmige Klänge ein. Kurz vor Schluss des Beispiels etwa - Dietrich arbeitet intensiv mit absteigenden Quintgängen - wird die hyperaeolische quinta deficiens $f a-f a\left(f^{-}-b\right)$ in der Mittelstimme geschickt durch die in der Obersexte parallel laufende Diskantquinte sol-ut $\left(d^{\prime \prime}-g^{\prime}\right)$ kaschiert, und ganz ähnlich wird die hyperaeolische Finalis $h$ in eine durch die Rahmenstimmen gebildete phrygische Klausel mit Finalis $e$ eingepasst (siehe Notenbeispiel 6). Dass die Parallelstimme zum Diskant als Cantus firmus und damit als modale Hauptstimme gelten soll, ist dem Satz nicht anzumerken.
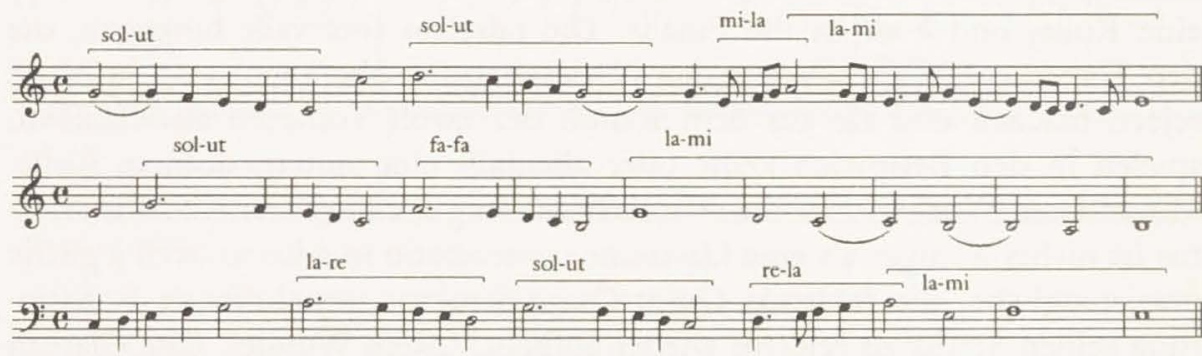

Notenbeispiel 6: Sixt Dietrich, Schluss des hyperaeolischen »O Domine Jesu Christe«

Noch weitaus drastischer setzt Dietrich in seiner ersten vierstimmigen Bearbeitung des Hyperphrygius, »Domine fac mecum $\aleph^{52}$, auf die Integration des Rejectus in Klänge. Schon die Entscheidung für eine Besetzung ad aequales, mit vier Stimmen in enger und tiefer Lage (die Schlüssel sind C3, C4, F4, F4), ist bezeichnend genug. Erschwert hat Dietrich das Verfolgen der einzelnen Stimmen zudem durch ständige Stimmkreuzungen, und außerdem hat er den Tenor in pausenlos durchlaufenden Semibreven vertont, auf jede Strukturierung, etwa durch Kadenzen, verzichtet und damit dem Modus eine entscheidende Möglichkeit der Profilierung genommen. Alle diese Maß-

50 Dod., S. 276f.; Übs. Miller, S. $348 f$.

51 S. Fuller, Defending the Dodecachordon (wie Anm. 47), S. 216, Anm. 62: »... while the tenor of the motet clearly exemplifies Glarean's Hyperaeolian, the cantus is closer to Hypophrygian, the bass to a mixture of Hypophrygian and Phrygian.«

52 Dod., S. 342f.; Übs. Miller, S. 421-423. 
nahmen zielen auf ein Verschmelzen der Stimmen zu einem kontinuierlichen Klangstrom, in dem zumal der Cantus firmus gänzlich aufgeht, gleich, ob es sich um wesentliche (siehe etwa in Notenbeispiel 7a in der 2. Akkolade das $f$, das als Terz im d-Klang verschwindet) oder unwesentliche Töne handelt. Bezeichnend ist auch der Schluss (Notenbeispiel 7b): Das Stück endet mit einer Sext-Oktav-Kadenz nach $\mathrm{G}$, dessen Oberterz $h$ der Cantus firmus beisteuert womit er satztechnisch wie eine Nebenstimme endet: als Altus.
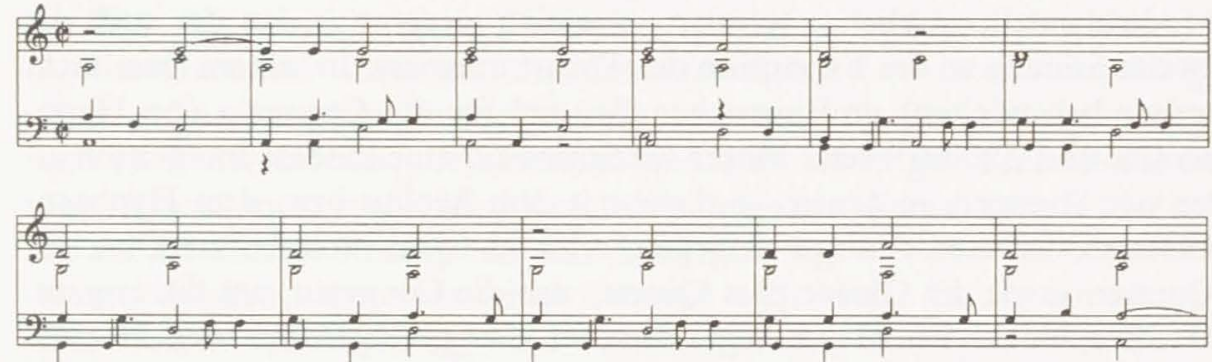

Notenbeispiel 7a: Sixt Dietrich, „Domine fac mecum« (ad aequales). Mittelabschnitt

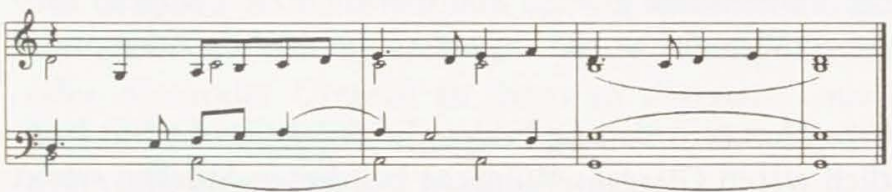

Notenbeispiel 7b: Sixt Dietrich, »Domine fac mecum« (ad aequales). Schlusstakte

Glarean war der Meinung, in Dietrichs Stück könne man die »natura « des Hyperphrygius aus dessen Phrasis und Finalis gut erkennen.. ${ }^{53}$ Damit schrieb er dem Modus eine Normalität zu, die seiner eigenen Theorie von einem Rejectus diametral widerspricht. Dietrich hat, wie es scheint, diesen Konflikt genau erkannt. Weil der Wunsch, einen Hyperphrygius zu komponieren, tatsächlich nur unter Preisgabe des Modus erfüllbar ist, wird in seinem Stück der Modus durch die Art seiner Gestaltung tatsächlich preisgegeben. Besser, kongenialer kann man sich die Realisierung von Glareans konträrem Procedere kaum vorstellen.

53 »... natura uero partim ex phrasi, partim finali claue facile pernoscatur. «: Dod., S. 342. 
Die Gestalt der von Glarean für das Dodekachordon bestellten mehrstimmigen Kompositionen hängt, wie sich zeigt, wesentlich von den Tonarten ab, die sie demonstrieren sollen. Dorius, Hypomixolydius und Phrygius, aber auch die Connexio von Aeolius und Hypoaeolius bieten wenig Probleme. Die modustypischen Intervalle und Ambitus sind grundsätzlich bekannt und vertraut, auch die Beschränkung auf den Cantus durus erfordert keinen besonderen kompositorischen Aufwand.

Meyers Kanons lassen erkennen, dass Glarean eine konsequent lineare Modusdarstellung ebenso tolerierte wie eine integrative, bei der auch die zweite Stimme an der Exposition der Tonart mitwirkt. In seinem (hier nicht weiter behandelten) umfangreichen Beispiel für die Connexio von Hypoaeolius und Aeolius ${ }^{54}$ setzt Meyer wiederum auf eine modale Integration aller vier Stimmen, in denen sämtliche mit dem Aeolius bzw. dem Hypoaeolius assoziierbaren Ambitus begegen, seien es leicht modifizierte Oktaven, Quinten sowie die Oktave plus Quarte, also die Connexio, um die es geht. Darüber hinaus hat Meyer angesichts der eher geringen modalen Herausforderung hier die Gelegenheit genutzt, sich nicht nur als modal versierter Vokalkomponist, sondern auch als Kenner der älteren mehrstimmigen Messkomposition zu profilieren; die beiden letzten Kyrie könnten überdies als seine ganz spezielle Visitenkarte gelten, weil hier mehrfach längere Ketten aus Semiminimen unüberhörbar die organistische Kolorierungspraxis anklingen lassen.

Eine weitaus größere Herausforderung bilden die Stücke im Lydius bzw. Hypolydius. Vermutlich waren Glareans Wünsche bei diesen Stücken schon erfüllt, wenn es gelang, die fatale Quinte $f a-f a$ ohne größere Einschränkungen zu realisieren - was Meyer nur partiell, Dietrich hingegen weitaus überzeugender schaffte. Die modale Prägung auch der übrigen Stimmen war angesichts dieses Problems eher zweitrangig; Hauptsache, die Musik verblieb möglichst durchweg im Cantus durus. Insgesamt vermitteln die meisten Stücke dieser Art, auch und gerade Meyers lydisch-jonische Doppelvertonung, einen eher angestrengten Eindruck. Damit korrespondieren sie exakt der angestrengten Manier, die Glareans Bemühungen eignet, den alten, wahren Lydius auf Kosten einer scheinbar verderbten Praxis immer wieder ins rechte Licht zu rücken.

Mit der Komposition derjenigen Modi, die Glarean wegen ihrer fehlenden Quint-Quart-Struktur ausdrücklich aus der Zahl regulärer Tonarten ausschloss, scheint die Auslieferung der Komponisten und ihrer Beispiele an

54 Dod., S. 402-417; Übs. Miller, S. 484-498. 
die Theorie endgültig über die Praktikabilität der Musik zu obsiegen. Aber Glarean hat sich hier selbst ad absurdum geführt. Einerseits weist er den Hyperaeolius wie den Hyperphrygius zurück, andererseits aber reizt es ihn, selbst diese modalen Monstrositäten praktisch zu demonstrieren - freilich unter Verzicht auf die Strukturen, denen sie ihren Status als Rejecti verdanken. Diesen Widerspruch hat Dietrich in zumindest einem seiner Beispiele perfekt auskomponiert. Modale Stimmigkeit geht in Klang auf, nur ein unhörbarer Modus rejectus, so der Komponist, ist ein wahrer Modus rejectus.

Wer von den bestellten Beispielen nähere Aufschlüsse über Glareans Modusverständnis - zumal in der Mehrstimmigkeit - erwartet, sieht sich getäuscht. Die Beispiele illustrieren, nicht anders als die übrigen Kompositionen im Dodekachordon, die einfachen und die schwierigen Bestandteile seiner Theorie, mehr nicht. Man kann vermuten, dass Glarean gerade hier gerne auf die modale Konzeption aller Stimmen hingewiesen hätte, aber selbst diesen Gefallen haben ihm weder Dietrich noch Meyer immer tun können. Der Nutzen der Stücke lag wohl vor allem in ihrer konzeptionellen Breite: Gerade die schwierigen Tonarten zwangen die Komponisten zu ganz unterschiedlichen Techniken ihrer Realisierung - bis zum Extrem ihrer bewussten Unterdrückung. Und vielleicht war es gerade der Reiz, auch das scheinbar Unmögliche zu versuchen, der - zusammen mit der gelehrten Aura, die das Dodekachordon verbreitete - später Komponisten wie Homer Herpol oder Alexander Utendal zu ihren an Glareans Tonartenlehre orientierten Motettensammlungen anregte und damit eine produktive Glarean-Rezeption recht eigentlich erst in Gang brachte. 